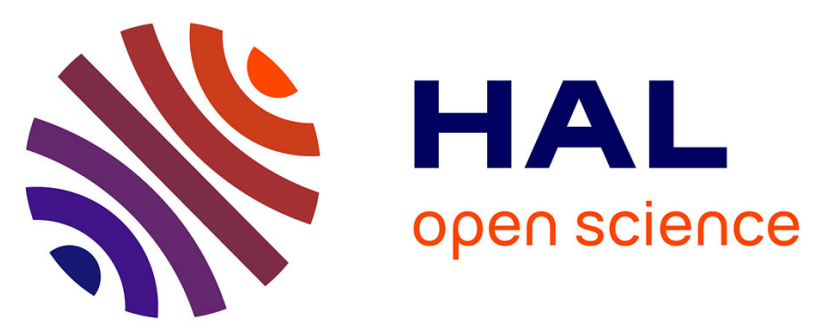

\title{
Acute redistribution of regional left ventricular work by cardiac resynchronization therapy determines long-term remodelling
}

\author{
Jürgen Duchenne, John M Aalen, Marta Cvijic, Camilla K Larsen, Elena
} Galli, Stéphanie Bézy, Ahmed S Beela, Serkan Ünlü, Efstathios D Pagourelias, Stefan Winter, et al.

\section{To cite this version:}

Jürgen Duchenne, John M Aalen, Marta Cvijic, Camilla K Larsen, Elena Galli, et al.. Acute redistribution of regional left ventricular work by cardiac resynchronization therapy determines longterm remodelling. European Heart Journal - Cardiovascular Imaging, 2020, 21 (6), pp.619-628. 10.1093/ehjci/jeaa003 . hal-02498461

HAL Id: hal-02498461

https://hal-univ-rennes1.archives-ouvertes.fr/hal-02498461

Submitted on 24 Mar 2020

HAL is a multi-disciplinary open access archive for the deposit and dissemination of scientific research documents, whether they are published or not. The documents may come from teaching and research institutions in France or abroad, or from public or private research centers.
L'archive ouverte pluridisciplinaire HAL, est destinée au dépôt et à la diffusion de documents scientifiques de niveau recherche, publiés ou non, émanant des établissements d'enseignement et de recherche français ou étrangers, des laboratoires publics ou privés. 


\section{Acute re-distribution of regional left ventricular work by cardiac resynchronization therapy determines long-term remodelling}

Duchenne et al. Acute redistribution of work determines remodelling in CRT

Jürgen Duchenne, $\mathrm{PhD}^{\mathrm{a}, \mathrm{b}}$, John M. Aalen, MD ${ }^{\mathrm{c}, \mathrm{d}, \mathrm{e}}$, Marta Cvijic, MD, PhD ${ }^{\mathrm{a}, \mathrm{b}}$, Camilla K. Larsen, $\mathrm{MD}^{\mathrm{c}, \mathrm{d}, \mathrm{e}}$, Elena Galli, MD, PhD f,g, Stéphanie Bézy, MSc ${ }^{\mathrm{a}, \mathrm{b}}$, Ahmed S. Beela, MD ${ }^{\mathrm{a}, \mathrm{b}}$, Serkan Ünlü, MD ${ }^{\mathrm{a}, \mathrm{b}}$, Efstathios D. Pagourelias, MD, PhD ${ }^{a, b}$, Stefan Winter, $M D^{h}$, Einar Hopp, MD, PhD', Erik Kongsgård, MD, PhD ${ }^{c, d}$, Erwan Donal, MD, PhD f,g, Wolfgang Fehske, MD, PhD ${ }^{h}$, Otto A. Smiseth, MD, PhD ${ }^{c, d, e^{*}}$, Jens-Uwe Voigt, $\mathrm{MD}, \mathrm{PhD}^{\mathrm{a}, \mathrm{b}, *}$

a) Department of Cardiovascular Sciences, KU Leuven, Leuven, Belgium

b) Department of Cardiovascular Diseases, University Hospitals Leuven, Leuven, Belgium

c) Institute for Surgical Research, Oslo University Hospital and University of Oslo, Oslo, Norway

d) Institute of Clinical Medicine, University of Oslo, Oslo, Norway

e) Department of Cardiology, Oslo University Hospital, Oslo, Norway

f) LTSI, Inserm 1099, University of Rennes, Rennes, France

g) Department of Cardiology, CHU Rennes, France

h) Klinik für Innere Medizin und Kardiologie, St. Vinzenz Hospital, Cologne, Germany

i) Division of Radiology and Nuclear Medicine, Oslo University Hospital, Oslo, Norway

Disclosures: All authors report no relationships that could be construed as a conflict of interest.

Corresponding author contact information:

Prof. Dr. Jens-Uwe Voigt

University Hospitals Leuven, Department of Cardiovascular Diseases

Herestraat 49, 3000 Leuven, Belgium

Tel.: $\quad+32 / 16 / 349016$

Fax: $\quad+32 / 16 / 344240$

E-mail: $\quad$ jens-uwe.voigt@uzleuven.be

*: Profs. Otto A. Smiseth and Jens-Uwe Voigt share last authorship. 


\section{ABSTRACT}

Aim: Investigating the acute impact of cardiac resynchronization therapy (CRT) on regional myocardial work distribution in the left ventricle (LV) and to which extent it is related to long-term reverse-remodelling.

Methods and results: One hundred thirty heart failure patients, referred for CRT implantation, were recruited in our prospective multicentre study. Regional myocardial work was calculated from noninvasive segmental stress-strain-loop-area before and immediately after CRT. The magnitude of volumetric reverse-remodelling was determined from the change in LV end-systolic volume (ESV), $11 \pm 2$ months after implantation. CRT caused acute re-distribution of myocardial work across the LV, with an increase in septal work, and decrease in LV lateral wall work (all $p<0.05$ ). Amongst all LV walls, the acute change in work in the septum and lateral wall of the four-chamber view correlated best and significantly with volumetric reverse-remodelling $(r=0.62, p<0.0001)$, with largest change seen in patients with most volumetric reverse-remodelling. In multivariate linear regression analysis, including conventional parameters such as pre-implant QRS morphology and duration, LV ejection fraction, ischemic origin of cardiomyopathy, and the re-distribution of work across the septal and lateral walls, the latter appeared as the strongest determinant of volumetric reverse-remodelling after CRT (model $\mathrm{R}^{2}=0.414, \mathrm{p}<0.0001$ ).

Conclusions: The acute re-distribution of regional myocardial work between the septal and lateral wall of the LV is an important determinant of reverse-remodelling after CRT-implantation. Our data suggest that treatment of the loading imbalance should therefore be the main aim of CRT.

Key words: cardiac resynchronisation therapy; myocardial work; dyssynchrony; left bundle branch block; remodeling. 


\section{INTRODUCTION}

Dyssynchronous left ventricular (LV) contraction, resulting from conduction delays such as left bundle branch block (LBBB), is associated with the development of LV systolic dysfunction and dilatation ${ }^{1,2}$. Animal models have demonstrated a re-distribution of myocardial work from the early to the late activated walls of the left ventricle in dyssynchronous ventricles ${ }^{3,4}$. Such animal studies also suggest, that the modification of workload distribution might be the trigger for reverse remodelling after re-synchronization of the heart.

However, clinical data supporting these findings are limited, as measuring regional myocardial work is technically challenging ${ }^{5}$. Segmental pressure-strain-loops may serve as a surrogate of myocardial work per volume-unit, but the necessary invasive LV pressure measurements ${ }^{4,6,7}$ remain a hurdle in clinical practice ${ }^{8,9}$. A methodology for a completely non-invasive approach was proposed and validated against invasive measurements by Russell et al ${ }^{10}$. While the use of pressure as surrogate of wall stress assumes a homogeneous wall stress distribution, the differential thickening and thinning in the remodelled hearts can be compensated by the use of stress-strain-loops, where both regional wall thickness and mid-wall curvature are taken into account ${ }^{11}$. The resulting non-invasive regional stress-strain-loops thereby provide a measure of regional myocardial work per volume-unit.

We have previously demonstrated long-term homogenisation of myocardial work distribution in patients with a clear volumetric response to cardiac resynchronisation therapy (CRT) ${ }^{11}$. We hypothesize that (I) the work is already re-distributed immediately after CRT-implantation, that (II) this re-distribution is the trigger for LV volumetric reverse-remodelling in these patients, and (III) that probably not all patients show the same level of work re-distribution.

In this prospective multicentre study, we therefore investigate the acute impact of CRT on regional myocardial work - measured as non-invasive regional stress-strain-loop-area - and determined how the re-distribution of regional loading by CRT is related to long-term volumetric reverse-remodelling. 


\section{METHODS}

\section{Study population}

One hundred thirty routine heart failure patients, referred for CRT, were recruited through the cardiology departments of the University Hospitals Leuven, Leuven, Belgium, the Oslo University Hospital, Oslo, Norway, and the St. Vinzenz Hospital, Cologne, Germany. The indication for CRT was determined according to the respective contemporary guidelines of the European Society of Cardiology ${ }^{12,13}$. Patients with atrial fibrillation were excluded. All patients were on optimal pharmacologic therapy for at least 3 months before CRT. Ischemic aetiology of cardiomyopathy was proven by coronary angiography, scintigraphy or magnetic resonance imaging. At the time of inclusion, none of the candidates required interventional or surgical treatment for coronary disease or valvular heart disease.

The study was approved by the respective local ethical committees, and informed consent was obtained from the study participants. The current manuscript uses in-part data of the WORK-CRT study (ClinicalTrials.gov Identifier NCT02537782).

\section{Echocardiography}

Patients underwent echocardiography within 1 week before CRT (Pre-CRT), within 1 week after CRT (Post-CRT), and after approximately 12 months of follow-up after CRT (Follow-up). Echocardiographic data were acquired using commercially available scanners (Vivid E9 and E95, GE Vingmed Ultrasound, Horten, Norway). Digitally stored data were analysed off-line using EchoPAC (version 202, GE Vingmed Ultrasound).

LV end-diastolic (EDV) and end-systolic volumes (ESV), and LV ejection fraction (LVEF) were measured using the modified biplane Simpson's method. Volumetric reverse-remodelling after CRT was assessed as the relative change in LV ESV at Follow-up ( $\Delta$ LV ESV). 
Two-dimensional speckle tracking was performed using images acquired in apical 4-chamber, 2chamber and 3-chamber views (frame rate $>60$ frames/s) at Pre-CRT and Post-CRT study time points. Eighteen LV segmental mid-wall longitudinal strain curves were analysed. Opening and closing artefacts on Doppler tracings of LV valves were used to determine time intervals ${ }^{14}$. Mid-segmental LV wall thickness was measured at end-diastole (defined by mitral valve closure), using the three apical views. Papillary muscles were not included in thickness measurements.

\section{Calculation of segmental myocardial work}

A dedicated, MATLAB-based (version 2017b, The MathWorks, Inc., Natick, MA, USA) research software (TVA version 22.00, JU Voigt, Leuven) was used to determine the segmental myocardial work by means of stress-strain-loop-area, as previously described ${ }^{11}$. In short, the non-invasive estimate of LV-pressure was based on the methodology of Russell et al ${ }^{10}$. LV segmental mid-wall curvature was dynamically estimated from full-trace export from EchoPAC and used together with the segmental wall thickness measurements and LV-pressure to estimate segmental wall stress according to the formula of Laplace ${ }^{15}$. Segmental stress-strain-loops were generated, the area of which was considered to represent myocardial work per volume-unit ${ }^{11,16}$. Myocardial work analysis was performed by one experienced observer, blinded to volumetric measurements.

Myocardial work per LV wall was calculated as average of the stress-strain-loop-areas of the basal, mid, and apical segments of the respective wall. The immediate effect of the CRT was calculated as the work difference between the Post-CRT and Pre-CRT time point (Post - Pre CRT work).

Furthermore, the difference between the work of the lateral and septal wall was calculated from the apical four-chamber view for every patient at the Pre-CRT and Post-CRT time point (Lateral-to-septal work difference). The decision to focus on these two walls was based on the results of a multivariate linear model, analysing the contribution of work change of each of the six LV walls to volumetric reverse-remodelling after CRT. 
Finally, to evaluate the immediate re-distribution of regional myocardial work caused by the CRTimplantation on the septal and lateral wall, we determined the change in the Lateral - septal work difference between the Post- and Pre-CRT time point ( $\Delta$ Lateral-to-septal work).

In order to better illustrate the relation between immediate changes in myocardial work and the degree of volumetric reverse-remodelling at follow-up, characteristics of patients in the lowest and highest quartile of volumetric reverse-remodelling were compared.

\section{Systolic strain difference}

Systolic strain was calculated as the difference of the end-systolic and end-diastolic longitudinal strain in the LV lateral and septal segments of the four-chamber view. Segmental values of both walls were then averaged to represent septal and lateral systolic strain. The difference in average systolic strain of both walls was then used as alternative indicator of regional functional imbalance.

\section{Cardiac resynchronisation therapy}

CRT-implantation was performed according to local standards of the contributing institution. In short, LV pacing leads were positioned, guided by coronary venography, preferably in the lateral and postero-lateral venous branches. After implantation, the device was set to bi-ventricular pacing in all patients. Device settings were optimised, as deemed clinically appropriate, before patients underwent the Post-CRT echocardiography, based on surface electrocardiogram (ECG) and/or Doppler echocardiography ${ }^{17}$.

\section{Statistical analysis}

Analysis was performed using SPSS Statistics 20 (IBM, Chicago, IL, USA) and MedCalc (MedCalc, Ostend, Belgium). All continuous variables are expressed as mean \pm standard deviation if normally distributed, otherwise by median \pm interquartile range. Normality was assessed using the ShapiroWilk test. Comparison among LV walls was performed using one-way ANOVA, with Bonferroni correction for repeated measures. Paired samples t-test or chi-squared test were used for 
comparison of continuous or categorical variables, respectively. The relation between variables was assessed by univariate linear regression analysis with Pearson's correlation coefficients. Z-statistics were used to compare correlation coefficients. Subsequently, contributors with a significant correlation in the univariate analysis were entered in a multiple linear regression model, using the backwards method, and after evaluation of possible collinearity. Intra- and inter-observer-variability was assessed using intra-class-correlation (ICC), with $95 \%$ confidence-interval (Cl). Statistical significance was set at a two-tailed probability level of $p<0.05$. 


\section{RESULTS}

\section{Clinical and echocardiographic characteristics}

Clinical characteristics of the study population are summarised in Table 1. The LV EDV, ESV, EF and GLS changed significantly at follow-up, compared to baseline (all $p<0.05$ ) (Table 2). The median $\Delta$ LV ESV after $11 \pm 2$ months of follow-up was $-28 \pm 26 \%$

The patient quartile with the most volumetric reverse-remodelling at follow-up (LV ESV reduction of more than $48 \%$ ) was characterised by significantly less men, less patients with ischemic cardiomyopathy, and with less dilated left ventricles compared to the patient quartile with the least volumetric reverse-remodelling (LV ESV reduction of less than 9\%) (all $p<0.05$ ) (Table 1). In the patient quartile with most volumetric reverse-remodelling, advantageous changes in LV volumes, LVEF and GLS were highly significant (all $p<0.0001$ ), while changes in the patient quartile with the least volumetric reverse-remodelling were all non-significant (Table 2).

\section{Myocardial work distribution}

\section{Before CRT implantation}

Before CRT, myocardial work was significantly different among the LV walls (Figure 1A) (ANOVA $p<0.0001$ ), with the lowest work observed in the septal wall, and highest work in the lateral wall. On average, the maximum work difference among walls was higher in the patient quartile with the most volumetric-response (3242 mmHg*\%; ANOVA among walls: $p<0.0001$ ), compared to the patient quartile with the least response (1132 $\mathrm{mmHg} \%$; ANOVA among walls: $p=0.01$ ) (Figure $2 \mathrm{~A}$ vs. 2D, respectively).

\section{After CRT implantation}

Immediately after CRT, the work distribution among the LV walls was on average homogeneous (ANOVA $p=0.122$ ) (Figure 1B). The largest increase in work was observed in the septal wall and the 
largest decrease in work in the lateral wall (Post - Pre CRT work) (Figure 1C). In the patient quartile with most volumetric-response, changes per wall were most pronounced (all $p<0.05$ ). In this patient group, we observed a reversed pattern of inhomogeneity after CRT (ANOVA $p=0.009$ ), with septal work being higher than in other LV walls (Figure 2B). This finding was significantly different to the patient quartile with least volume-response $(\mathrm{p}<0.05)$ (Figure $2 \mathrm{~B}$ vs. $2 \mathrm{E})$. In these patients, no relevant changes in work distribution were observed between Pre- and Post-CRT (all p=N.S.) (Figure 2D, E, F).

\section{Relation between acute change in myocardial work and volumetric reverse-}

\section{remodelling}

The acute change in myocardial work per LV wall showed significant correlation to reverseremodelling after CRT ( $\triangle$ LV ESV) for the septal and lateral wall (all $p<0.01$ ) (Figure 3). Multivariate linear regression analysing the different apical views revealed that the Post - Pre CRT work difference of the four chamber view (infero-septal and antero-lateral walls) explained the highest percentage of LV ESV change after CRT $\left(R^{2}=0.372, p<0.0001\right)$.

For the entire study cohort, the Lateral-to-septal work difference decreased from $2130 \mathrm{mmHg} \%$ pre-CRT to $-95 \mathrm{mmHg} * \%$ post-CRT $(\mathrm{p}<0.0001)$ (Figure $4 \mathrm{~A})$. Furthermore, the acute change in Lateralto-septal work difference caused by CRT ( $\Delta$ Lateral - septal work) was significantly larger in the patient quartile with the most volumetric-response compared to the one with the least response $(p<0.01)$ (Figure 4B).

The acute change in Lateral-to-septal work difference ( $\Delta$ Lateral - septal work) caused by CRT was strongly and significantly related to the long term volumetric-response of the $\operatorname{LV}(r=0.62, p<0.0001$, Figure 5). A similar, but slightly weaker correlation was found for the pre-CRT Lateral-to-septal work difference alone $(r=0.44, p<0.0001$; Supplemental Figure 1) $(p=0.044$ between both correlation coefficients). 
Multivariate linear regression analysis including the pre-CRT lateral-to-septal work difference, QRSmorphology, QRS-duration, LVEF, and ischemic origin of cardiomyopathy, showed that only lateralto-septal work difference and ischemic origin of cardiomyopathy are significant determinants of volumetric reverse-remodelling ( $p<0.0001$ and $p=0.018$, respectively) (model $R^{2}=0.269$ ) (Table 3 ).

However, a multivariate model with the same parameters, but with $\Delta$ lateral - septal work instead of pre-CRT Lateral-to-septal work difference, did not show a significant influence of ischemic origin of cardiomyopathy. Only $\Delta$ lateral - septal work was a significant determinant of volumetric reverseremodelling $(p<0.0001)$ (model $\left.R^{2}=0.414\right)$ (Table 4).

\section{Systolic strain difference}

The septal and lateral systolic strain followed a comparable pattern as myocardial work, with lowest systolic strain observed in the septal wall, and highest systolic strain in the lateral wall before CRT, and a relative homogenisation after CRT (Supplemental Figure 2 and 3).

For the entire study cohort, the Lateral-to-septal strain difference decreased from $-6.51 \%$ to $-1.05 \%$ (Supplemental Figure 4A). Furthermore, the acute change in Lateral-to-septal strain difference caused by CRT ( $\Delta$ Lateral-to-septal strain) was significantly larger in the patient quartile with the most volumetric-response compared to the one with the least response $(p<0.01)$ (Supplemental Figure 4B), and correlated significantly to the long-term volumetric response of the LV ( $r=0.55$, $p<0.0001$ ) (Supplement Figure 5).

\section{Reproducibility}

LV stress-strain-loop-area showed good intra-observer (ICC: $0.805,95 \% \mathrm{CI}(0.757-0.846)$ and interobserver agreement (ICC: $0.906,95 \% \mathrm{CI}(0.884-0.923)$. 


\section{DISCUSSION}

\section{Main findings}

The present multicentre study demonstrates that a significant re-distribution of regional myocardial work from the LV lateral wall to the septum occurs immediately after CRT-implantation. The most pronounced acute changes, leading to an overshooting loading of the septum, were observed in the patients who showed the most long-term volumetric reverse-remodelling. Patients with the least long term reverse-remodelling or deterioration showed no acute changes in workload distribution after CRT-implantation. Among parameters such as QRS-morphology, QRS-duration, LVEF, ischemic origin of cardiomyopathy and the acute re-distribution of regional myocardial work, the latter was found to be the strongest determinant of long-term volumetric reverse-remodelling after CRT.

\section{Distribution of regional myocardial work before CRT}

Before CRT, the distribution of regional myocardial work is heterogeneous (Figure 1A, 2A, and 2D). In the patient quartile with the best volume response (Figure 2A), we found the most pronounced and significant difference in regional myocardial work between the septal and lateral walls. The regional differences were statistically significant, and the findings in-line with previous observations from experimental animal studies where dyssynchronous LV activation resulted in un-loading of the early activated regions and higher load in the late activated regions ${ }^{3,4,10}$. The good response to CRT in this patient group is likely related to the fact that the electrical conduction delay lead to strong inhomogeneity in myocardial work, which can be corrected by CRT.

In contrast, the patient quartile with least volumetric response showed a less pronounced pattern of heterogeneous work distribution before CRT-implantation (Figure 2D), despite the presence of equally wide QRS. This finding might be attributed to significantly higher scar burden with varying localization in this group, as scar cannot be influenced by pacing. Presenting on average less negative septal work (i.e. less "wasted" work) implies also, that the potential to improve septal function is 
reduced. At the same time, the lateral wall is exposed to less pre-stretch and performs therefore less work, which explains that here the potential for unloading by CRT is lower ${ }^{18,19}$.

\section{Acute changes in regional myocardial work distribution with CRT}

CRT-device implantation leads acutely to an increase in septal work and a decrease in lateral work with on average homogeneous regional myocardial work distribution (Figure 1). With this, work distribution is more homogeneous as both walls contract during the pressure rise in the ventricle during early systole.

Interestingly, immediately after CRT, the septum showed highest myocardial work in the patient group with most volumetric response, exceeding the work of the other LV walls (Figure 2). Before CRT, the septum bears low load and is thin, while the lateral wall bears a high load and is thick. This maladaptation is then reversed by implantation of the CRT device, and the reversal of workload distribution with a relative overload of the septum and an under-load of the lateral wall then becomes the driving force of wall thickness normalisation during reverse-remodelling ${ }^{11,16}$. In contrast, in the patient quartile with least volume response, no relevant changes in work distribution were observed immediately after CRT-implantation (Figure 2).

Acute work re-distribution from the lateral to the septal wall was predominantly related to longterm reverse remodelling while no relevant anterior-inferior redistribution was observed (Figure 3). This is in agreement with the function of CRT which corrects a septal-lateral activation delay ${ }^{11}$, and with the repeated observations that parameters indicating mechanical dyssynchrony in septal-lateral direction are related to CRT success ${ }^{11,20-22}$.

\section{Regional work distribution and reverse remodelling}

The present study demonstrates that the distribution or regional myocardial work and - even more - the acute re-distribution of regional work are important determinants for the long-term volumetric reverse-remodelling after CRT-implantation. 
Furthermore, the predictive value of work re-distribution was dominant, even when ischemic aetiology of cardiomyopathy was taken into account. Adding information on ischemic aetiology to myocardial work mattered only before CRT, which indicates that work heterogeneity found before CRT is not only the result of loading differences which can be treated by resynchronization, but has likely also an ischemic component. Indeed, total myocardial scar burden has been shown before to be associated with non-response to $\mathrm{CRT}^{23}$, and a LBBB-pattern of work distribution may be mimicked by a septal scar, which also reduces septal work ${ }^{19}$. In such hearts, successful redistribution of myocardial work may not be possible by means of CRT which, however, becomes only overt after CRT-implantation if scar is not recognized. Prediction of CRT response by work analysis before implantation may therefore require an accompanying myocardial viability analysis. The fact that ischemic aetiology disappears as an independent variable from the multivariate model with the acute change in work, may indicate that ischemic damage is already reflected in a (reduced) redistribution of myocardial work.

Ultimately, analysis of the re-distribution of work seems to be a strong marker of a successful CRTtreatment.

\section{Systolic strain difference}

In this study, changes in systolic strain and myocardial work paralleled each other. Given that regional systolic strain is a main determinant of regional myocardial work, it can be expected that both parameters reflect the same phenomena similarly. We noted, however, a slightly weaker correlation of simple systolic strain measurements to volumetric response which might be due to the fact that our work calculation also considered the differential segmental wall thickness and curvature. This is in contrast to current commercial software which uses the same pressure estimates in all segments. 


\section{Clinical implications}

Our data suggest that CRT candidate selection could benefit from regional myocardial work assessment. Conventional pre-CRT characteristics such as pre-implant QRS-morphology, QRSduration, and LVEF on the other hand, failed to add significant value.

However, volumetric reverse-remodelling after CRT relates significantly stronger to the acute redistribution of regional work than to the pre-CRT regional work distribution. Our data therefore suggest that successful CRT is not only reflected by having potentially curable loading inhomogeneities before implantation, but more importantly by its capacity to successfully recruit and homogenize these loading inhomogeneities, irrespective of the presence of ischemic cardiomyopathy. Therefore, treatment of the work imbalance is the main prerequisite to a successful re-synchronization and volumetric reverse-remodelling and should therefore be the main aim of CRT. Other parameters, which also directly reflect loading inhomogeneities and include the effect of scar, such as apical rocking and septal flash may also have this advantage ${ }^{24}$.

\section{Study limitations}

LV pressure, and with this, regional wall stress estimates were based on assumptions of LV pressure changes during the cardiac cycle ${ }^{9-11,16}$. Previous studies have shown, however, that these assumptions, although inappropriate for a more detailed shape analysis of pressure- or stress-strainloops, are sufficiently reliable for the context of regional work analysis ${ }^{9,10}$.

The magnitude of volumetric reverse-remodelling can only be a surrogate parameter for CRT success. However, we avoided dividing our study group in "responders" and "non-responders" based on volume changes but rather compared our findings in work distribution to reverse remodelling on a continuous scale. Nevertheless, a longer follow-up after CRT and the assessment of survival data and clinical end-points could potentially provide an even more complete image. 
We used myocardial work as a comprehensive and intuitive tool to measure and compare the work load in different parts of the left ventricle. To which extent this tool is superior to other indices of mechanical dyssynchrony in the clinic remains to be proven in future studies. E.g., future studies should investigate whether workload distribution in LV dyssynchrony shows close relation to signs of mechanical dyssynchrony, such as apical rocking and septal flash.

\section{CONCLUSIONS}

To our knowledge, this is the first study exploring the acute effect of CRT on regional myocardial work distribution. Previous studies have either only investigated myocardial work before CRT ${ }^{25}$ and/or during follow-up when volumetric reverse-remodelling was evaluated ${ }^{11,26}$. Our findings demonstrate, that the acute re-distribution of work is strongly related to long-term reverseremodelling after CRT, (Figure 6). Our data therefore suggest that the re-distribution of regional myocardial loading is the underlying mechanism of a successful resynchronization therapy, which emphasizes the importance of considering myocardial mechanics and loading conditions in CRTpatients.

\section{ACKNOWLEDGMENTS}

The authors wish to thank Drs. Filipa Cordeiro, Monica Dobrovie, Aniela Petrescu and nurse Joris Vermunicht for assisting with echocardiography.

\section{FUNDING SOURCES}

This work was supported by a KU Leuven research grant [OT/12/084] and the Oslo Center for Cardiological Innovation. Individual research support came from the Research Foundation - Flanders (J-U.V.), Egyptian Ministry of Higher Education (A.S.B.), European Association of Cardiovascular 
Imaging (M.C., S.U., E.D.P.), South-Eastern Norway Regional Health Authority (C.K.L.) and the Norwegian Health Association (J.M.A), respectively. 


\section{REFERENCES}

[1] Vernooy K, Verbeek XA, Peschar M, Crijns HJ, Arts T, Cornelussen RN, et al. Left bundle branch block induces ventricular remodelling and functional septal hypoperfusion. Eur Heart J 2005;26:91-8.

[2] van Oosterhout M, Prinzen FW, Arts T, Schreuder JJ, Vanagt WY, Cleutjens JP, et al. Asynchronous electrical activation induces asymmetrical hypertrophy of the left ventricular wall. Circulation 1998;98:588-95.

[3] Duchenne J, Claus P, Pagouralias ED, Mada RO, Van Puyvelde J, Vunckx K, et al. Sheep can be used as animal model of regional myocardial remodelling and controllable work. Cardiol J 2018. doi:10.5603/CJ.a2018.0007. [E-pub ahead of print].

[4] Prinzen FW, Hunter WC, Wyman BT, McVeigh ER. Mapping of regional myocardial strain and work during ventricular pacing: experimental study using magnetic resonance imaging tagging. J Am Coll Cardiol 1999;33:1735-42.

[5] Voigt J-U. Global myocardial function, regional myocardial function, and the Daemon of Laplace. Eur Heart J Cardiovasc Imaging 2016;17:633-4.

[6] Urheim S, Rabben SI, Skulstad H, Lyseggen E, Ihlen H, Smiseth OA. Regional myocardial work by strain Doppler echocardiography and LV pressure: a new method for quantifying myocardial function. Am J Physiol Heart Circ Physiol 2005;288:2375-80.

[7] Delhaas T, Arts T, Prinzen FW, Reneman RS. Regional fibre stress-fibre strain area as an estimate of regional blood flow and oxygen demand in the canine heart. J Physiol 1994;477:481-96.

[8] Zweerink A, de Roest GJ, Wu L, Nijveldt R, de Cock CC, van Rossum AC, et al. Prediction of Acute Response to Cardiac Resynchronization Therapy by Means of the Misbalance in Regional Left Ventricular Myocardial Work. J Card Fail 2016;22:133-42.

[9] Hubert A, Le Rolle V, Leclercq C, Galli E, Samset E, Casset C, et al. Estimation of myocardial work from pressure-strain loops analysis: an experimental evaluation. Eur Hear J - Cardiovasc Imaging 2018;19:1372-9.

[10] Russell K, Eriksen M, Aaberge L, Wilhelmsen N, Skulstad H, Remme EW, et al. A novel clinical method for quantification of regional left ventricular pressure-strain loop area: a non-invasive index of myocardial work. Eur Heart J 2012;33:724-33. 
[11] Cvijic M, Duchenne J, Ünlü S, Michalski B, Aarones M, Winter S, et al. Timing of myocardial shortening determines left ventricular regional myocardial work and regional remodelling in hearts with conduction delays. Eur Heart J Cardiovasc Imaging 2018;19:941-9.

[12] Brignole M, Auricchio A, Baron-Esquivias G, Bordachar P, Boriani G, Breithardt O-A, et al. 2013 ESC guidelines on cardiac pacing and cardiac resynchronization therapy: the task force on cardiac pacing and resynchronization therapy of the European Society of Cardiology (ESC). Europace 2013;15:1070-118.

[13] Ponikowski P, Voors AA, Anker SD, Bueno H, Cleland JGF, Coats AJS, et al. 2016 ESC Guidelines for the diagnosis and treatment of acute and chronic heart failure: The Task Force for the diagnosis and treatment of acute and chronic heart failure of the European Society of Cardiology (ESC). Eur Heart J 2016;37:2129-200.

[14] Mada RO, Lysyansky P, Daraban AM, Duchenne J, Voigt J-U. How to define end-diastole and end-systole?: Impact of timing on strain measurements. JACC Cardiovasc Imaging 2015;8:148-57.

[15] Westerhof N, Stergiopulos N, Noble MIM. Snapshots of Hemodynamics 2010;2:45-8.

[16] Duchenne J, Turco A, Ünlü S, Pagourelias ED, Vunckx K, Degtiarova G, et al. Left ventricular remodelling results in homogenisation of myocardial work distribution. Circ Arrhythmia Electrophysio/ 2019. doi:10.1161/CIRCEP.118.007224. [E-pub ahead of print].

[17] Vardas PE, Auricchio A, Blanc J-J, Daubert J-C, Drexler H, Ector H, et al. Guidelines for cardiac pacing and cardiac resynchronization therapy. Europace 2007;9:959-98.

[18] Lumens J, Leenders GE, Cramer MJ, De Boeck BWL, Doevendans PA, Prinzen FW, et al. Mechanistic evaluation of echocardiographic dyssynchrony indices: patient data combined with multiscale computer simulations. Circ Cardiovasc Imaging 2012;5:491-9.

[19] Aalen JM, Remme EW, Larsen CK, Andersen OS, Krogh M, Duchenne J, et al. Mechanism of Abnormal Septal Motion in Left Bundle Branch Block: Role of Left Ventricular Wall Interactions and Myocardial Scar. JACC Cardiovasc Imaging 2019. doi:10.1016/J.JCMG.2018.11.030. [E-pub ahead of print].

[20] Szulik M, Tillekaerts M, Vangeel V, Ganame J, Willems R, Lenarczyk R, et al. Assessment of apical rocking: a new, integrative approach for selection of candidates for cardiac resynchronization therapy. Eur J Echocardiogr 2010;11:863-9.

[21] Stankovic I, Prinz C, Ciarka A, Daraban AM, Kotrc M, Aarones M, et al. Relationship of visually 
assessed apical rocking and septal flash to response and long-term survival following cardiac resynchronization therapy (PREDICT-CRT). Eur Hear J - Cardiovasc Imaging 2016;17:262-9.

[22] van der Bijl P, Khidir MJH, Leung M, Yilmaz D, Mertens B, Ajmone Marsan N, et al. Reduced left ventricular mechanical dispersion at 6 months follow-up after cardiac resynchronization therapy is associated with superior long-term outcome. Hear Rhythm 2018;15:1683-9.

[23] Ypenburg C, Roes SD, Bleeker GB, Kaandorp TAM, de Roos A, Schalij MJ, et al. Effect of Total Scar Burden on Contrast-Enhanced Magnetic Resonance Imaging on Response to Cardiac Resynchronization Therapy. Am J Cardiol 2007;99:657-60.

[24] Steelant B, Stankovic I, Roijakkers I, Aarones M, Bogaert J, Desmet W, et al. The Impact of Infarct Location and Extent on LV Motion Patterns. JACC Cardiovasc Imaging 2016;9:655-64.

[25] Galli E, Leclercq C, Hubert A, Bernard A, Smiseth OA, Mabo P, et al. Role of myocardial constructive work in the identification of responders to CRT. Eur Hear J - Cardiovasc Imaging 2018;19:1010-8.

[26] Vecera J, Penicka M, Eriksen M, Russell K, Bartunek J, Vanderheyden M, et al. Wasted septal work in left ventricular dyssynchrony: a novel principle to predict response to cardiac resynchronization therapy. Eur Heart J Cardiovasc Imaging 2016;17:624-32. 
Table 1: Clinical characteristics of the study population.

\begin{tabular}{|c|c|c|c|c|}
\hline Age (years) & $69 \pm 11$ & $66 \pm 13$ & $70 \pm 8$ & \\
\hline Male (\%) & $90(69)$ & $29(80)$ & $16(50)$ & $\dagger$ \\
\hline Ischemic cardiomyopathy (\%) & $52(40)$ & $18(50)$ & $5(16)$ & $\dagger$ \\
\hline Diabetes (\%) & $33(25)$ & $9(26)$ & $6(19)$ & \\
\hline Arterial hypertension (\%) & $89(72)$ & $21(59)$ & $24(74)$ & \\
\hline $\mathrm{DBP}(\mathrm{mmHg})$ & $69 \pm 11$ & $66 \pm 7$ & $70 \pm 14$ & \\
\hline B-blocker (\%) & $117(90)$ & $32(91)$ & $29(91)$ & \\
\hline ACEi/ARB (\%) & $122(94)$ & $31(89)$ & $33(100)$ & \\
\hline Spironolactone (\%) & $65(50)$ & $15(43)$ & $21(66)$ & \\
\hline
\end{tabular}




\begin{tabular}{c|c|c|c|c}
\hline Electrocardiographic parameters & & & \\
\hline LBBB (\%) & $99(76)$ & $24(69)$ & $29(91)$ & $167 \pm 20$ \\
\hline QRS duration (ms) & $165 \pm 21$ & $168 \pm 21$ & & \\
\hline
\end{tabular}

$A C E i=$ angiotensin-converting-enzyme inhibitor; $A R B=$ angiotensin-Il receptor blocker; $D B P=$ diastolic blood pressure; $L B B B=$ left bundle branch block; NYHA $=$ New York Heart Association; SBP $=$ systolic blood pressure. $(\dagger)=p<0.05$ between patients with LV ESV reduction of less than $9 \%$ versus $L V$ ESV reduction of more than $48 \%$. 
Table 2: Echocardiographic parameters of the study population.

\begin{tabular}{|c|c|c|c|c|}
\hline & $\begin{array}{l}\text { Entire population } \\
\qquad(n=129)\end{array}$ & $\begin{array}{l}\text { Reduction LV ESV less than } 9 \% \\
\qquad(n=36)\end{array}$ & $\begin{array}{l}\text { Reduction LV ESV more than } 48 \% \\
\qquad(n=33)\end{array}$ & Significance \\
\hline \multicolumn{5}{|c|}{ Pre-CRT: Echocardiographic parameters } \\
\hline LV EDV (ml) & $200 \pm 83$ & $229 \pm 104$ & $183 \pm 77$ & $\dagger$ \\
\hline LV ESV (ml) & $144 \pm 67$ & $165 \pm 83$ & $136 \pm 65$ & \\
\hline $\operatorname{LVEF}(\%)$ & $30 \pm 7$ & $29 \pm 7$ & $28 \pm 7$ & \\
\hline GLS (\%) & $-9 \pm 3$ & $-8 \pm 3$ & $-9 \pm 3$ & \\
\hline \multicolumn{5}{|c|}{ Post-CRT: Echocardiographic parameters } \\
\hline LV EDV (ml) & $194 \pm 82$ & $229 \pm 101$ & $172 \pm 70$ & $\dagger$ \\
\hline LV ESV (ml) & $137 \pm 65$ & $164 \pm 83$ & $131 \pm 53$ & \\
\hline $\operatorname{LVEF}(\%)$ & $30 \pm 7$ & $30 \pm 7$ & $30 \pm 7$ & \\
\hline GLS (\%) & $-9 \pm 3$ & $-8 \pm 3$ & $-9 \pm 3$ & \\
\hline \multicolumn{5}{|c|}{ Follow-up: Echocardiographic parameters } \\
\hline LV EDV (ml) & $159 \pm 84(\#)$ & $232 \pm 95$ & $101 \pm 40(\#)$ & $\dagger$ \\
\hline LV ESV (ml) & $104 \pm 71(\#)$ & $172 \pm 86$ & $52 \pm 25$ (\#) & + \\
\hline
\end{tabular}




\begin{tabular}{l|c|c|c|c}
\hline $\operatorname{LVEF}(\%)$ & $40 \pm 11(\#)$ & $30 \pm 8$ & $50 \pm 7(\#)$ & + \\
\hline GLS (\%) & $-12 \pm 4(\#)$ & $-8 \pm 5$ & $-15 \pm 3(\#)++$ \\
\hline
\end{tabular}

$E D V=$ end-diastolic volume; $E S V=$ end-systolic volume; $G L S=$ global longitudinal strain; $L V=$ left ventricular; $L V E F=$ left ventricular ejection fraction. (\#) = $p<0.05$ between pre-CRT and follow-up. $(\dagger)=p<0.05$ between patients with LV ESV reduction of less than $9 \%$ versus $L V$ ESV reduction of more than $48 \%$. 
Table 3: Multivariable linear regression analysis with left ventricular end-systolic volume reduction as dependent variable $\left(R^{2}=0.269\right)$.

\begin{tabular}{|c|c|c|c|}
\hline Regression variable & Beta & $95 \% \mathrm{Cl}$ & p-value \\
\hline QRS-morphology & 0.006 & $-11.08-10.42$ & 0.951 \\
\hline QRS-duration & 0.030 & $-0.160-0.234$ & 0.713 \\
\hline LV EF & 0.074 & $-0.311-0.820$ & 0.374 \\
\hline Ischemic cardiomyopathy & 0.201 & $1.834-19.35$ & 0.018 \\
\hline Lateral-to-septal work difference pre-CRT & -0.439 & $-0.008--0.003$ & 0.0001 \\
\hline
\end{tabular}


Table 4: Multivariable linear regression analysis with left ventricular end-systolic volume reduction as dependent variable $\left(R^{2}=0.414\right)$.

\begin{tabular}{|c|c|c|c|}
\hline Regression variable & Beta & $95 \% \mathrm{Cl}$ & p-value \\
\hline QRS-morphology & 0.076 & $-5.062-14.15$ & 0.351 \\
\hline QRS-duration & 0.086 & $-0.075-0.285$ & 0.251 \\
\hline LV EF & 0.056 & $-0.318-0.706$ & 0.455 \\
\hline Ischemic cardiomyopathy & 0.140 & $-0.521-15.35$ & 0.067 \\
\hline$\Delta$ Lateral-to-septal work & 0.624 & $0.005-0.009$ & 0.0001 \\
\hline
\end{tabular}




\section{FIGURES}

A Pre CRT

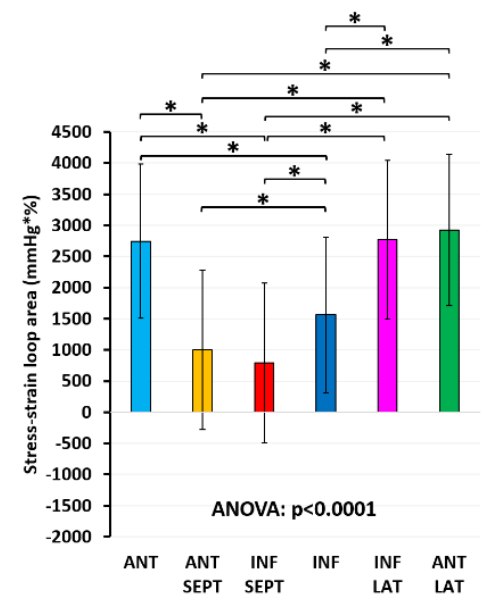

B

Post CRT

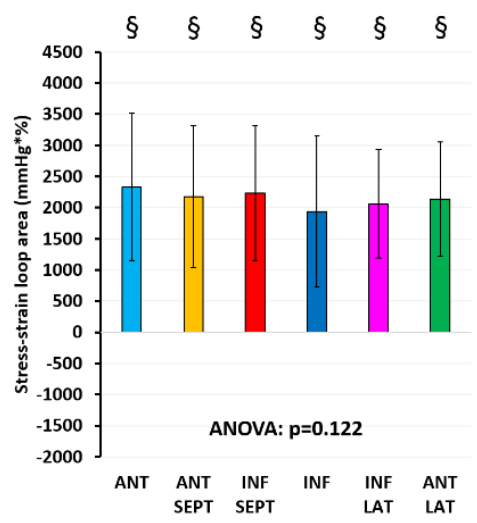

C Post - Pre CRT

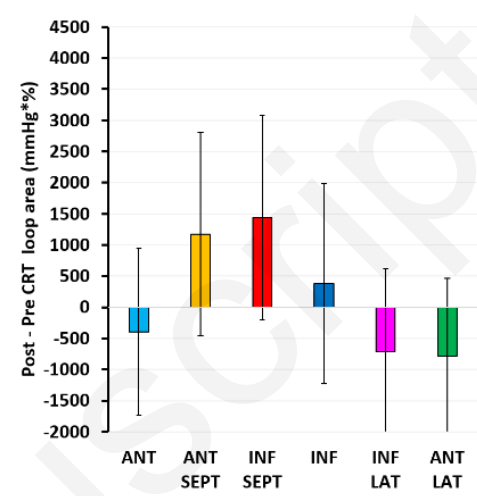

Figure 1: Distribution of myocardial work of the entire study population. Pre-CRT (A), Post-CRT (B), and difference between Post- and Pre-CRT (C). Colour coded bars represent the six LV walls. Significant differences are denoted with symbols: an * between walls, and a § between Pre-CRT vs. Post-CRT. ANT=anterior; ANT SEPT=antero-septum; INF SEPT=infero-septum; INF=inferior; INF LAT=infero-lateral; ANT LAT=antero-lateral. 
A

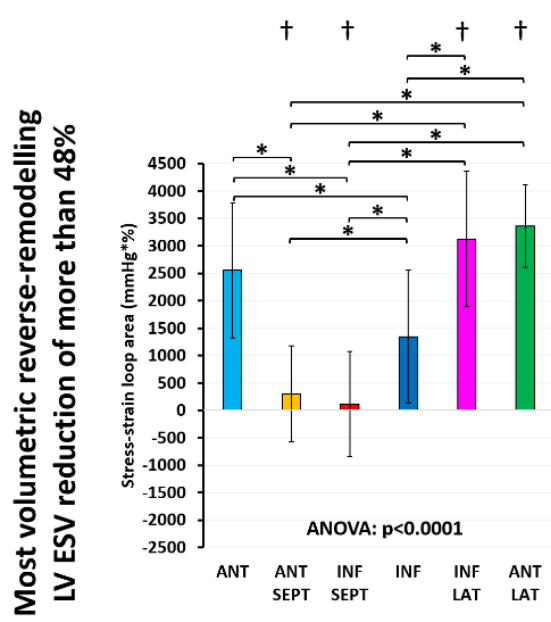

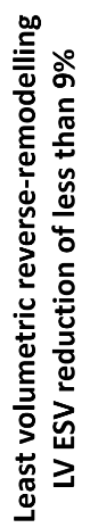

B

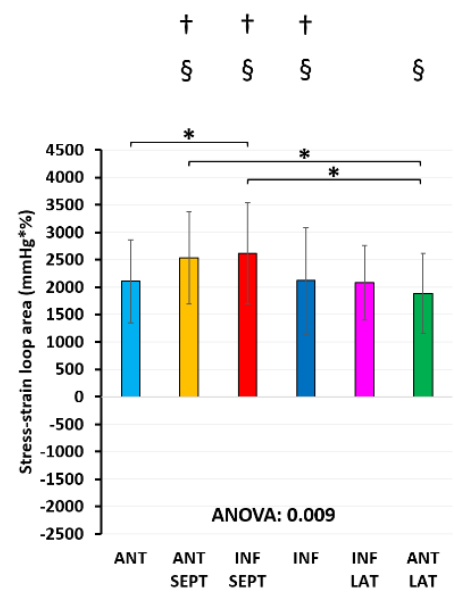

E

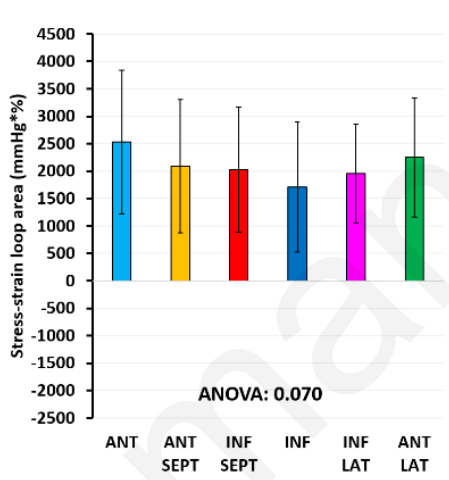

C

Post - Pre CRT

$+\quad+\quad+$
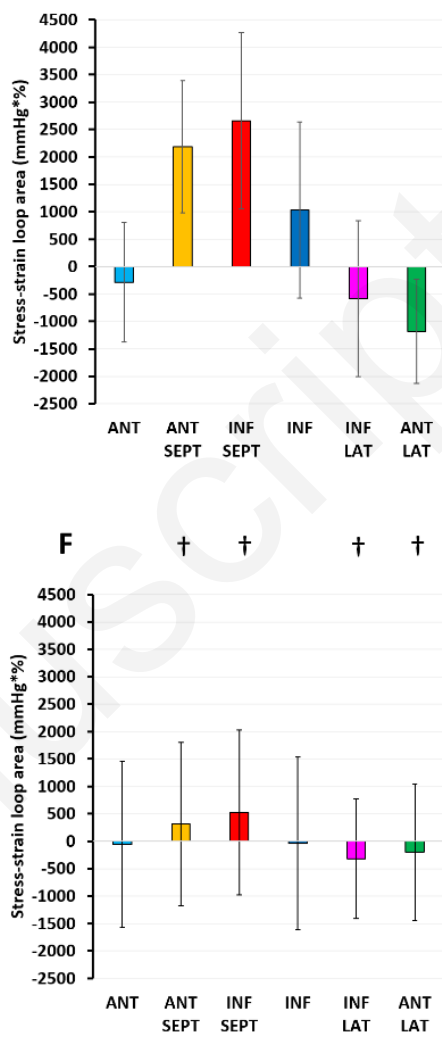

Figure 2: Distribution of myocardial work in patients with least and most volumetric reverseremodelling. Pre-CRT (left panels), Post-CRT (middle panels), and difference between Post- and PreCRT (right panels). Patients with most (LV ESV reduction of more than 48\%) or least (LV ESV reduction of less than 9\%) volumetric reverse-remodelling, in respectively upper $(A, B, C)$ and lower panels (D, E, F). Significant differences are denoted with symbols: an * between walls, and a $\S$ between Pre-CRT vs. Post-CRT, and a † between both patients groups. Annotations as in Figure 1. 

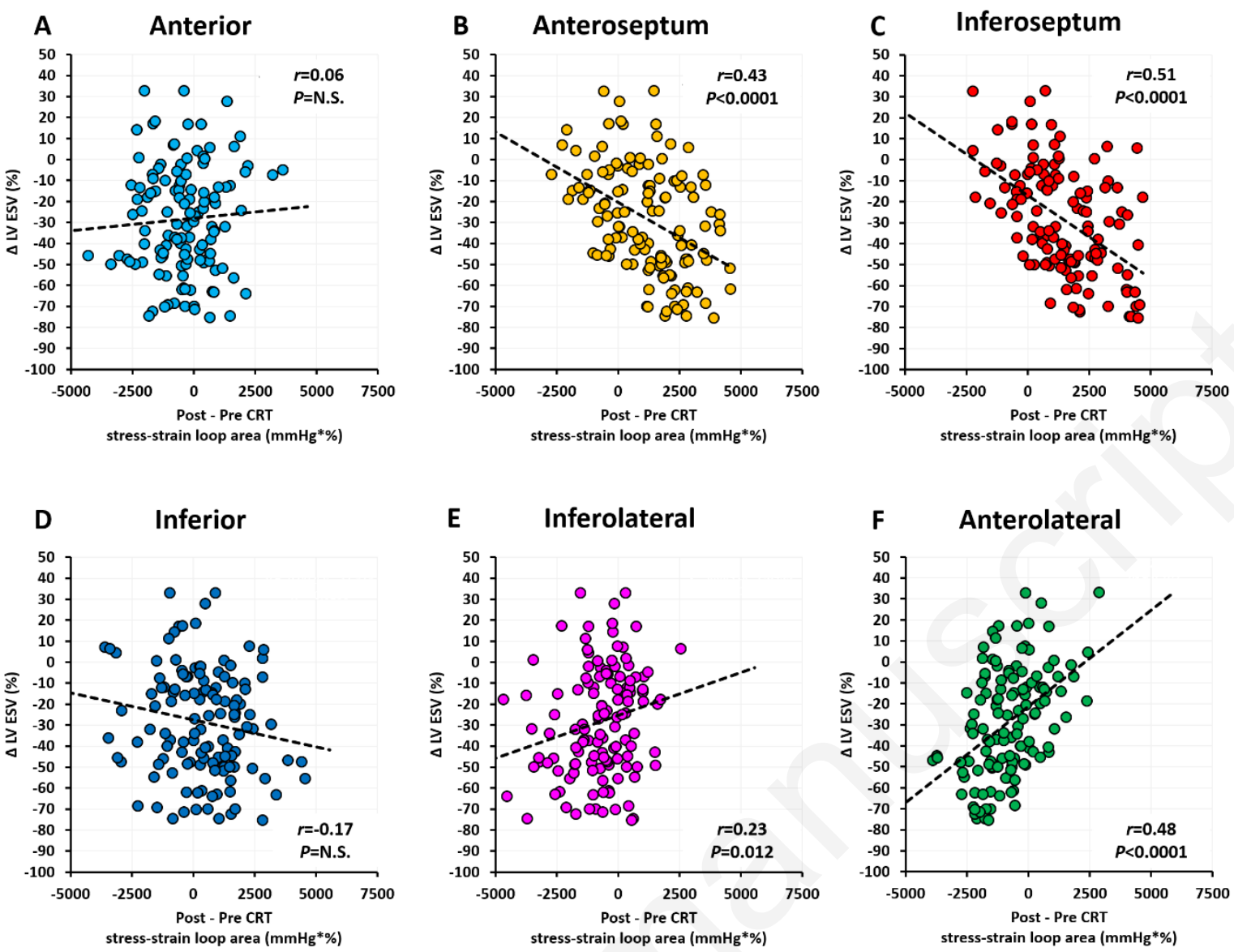

Figure 3: Correlation plots of the difference in Post - Pre CRT work per wall, and decrease in LV

ESV. Colour coded markers represent the specific LV wall: anterior (A), antero-septum (B), inferoseptum (C), inferior (D), infero-lateral (E), antero-lateral (F). 


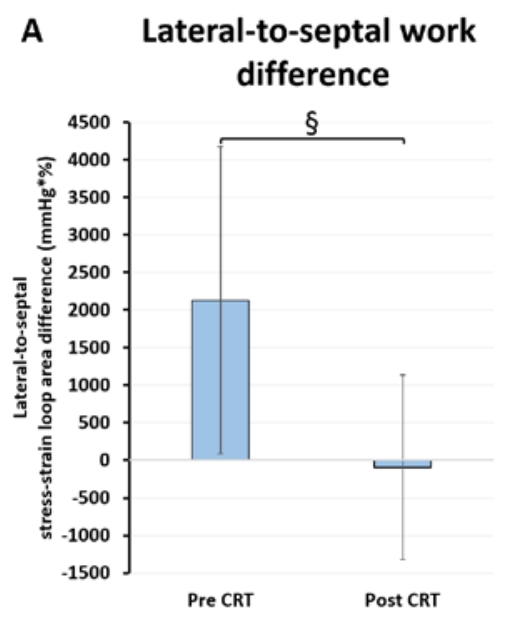

B $\Delta$ Lateral-to-septal work

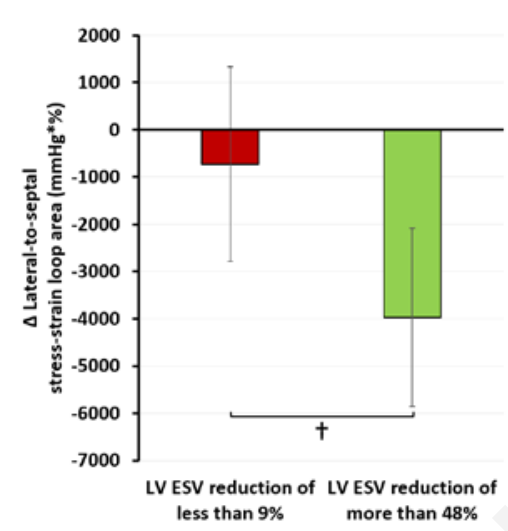

Figure 4: Lateral-to-septal work difference before and after CRT, and $\Delta$ lateral-to-septal work in patients with least and most volumetric reverse-remodelling. Panel A: Lateral-to-septal work difference. $\S=p<0.05$ between pre-CRT vs. post-CRT. Panel B: Acute change in Lateral-to-septal work difference caused by CRT ( $\Delta$ Lateral-to-septal work difference). Coloured bars represent patients with least (LV ESV reduction of less than 9\%; red bar) or most (LV ESV reduction of more than $48 \%$; green bar) volumetric reverse-remodelling. $\dagger=p<0.05$ between both patient groups.

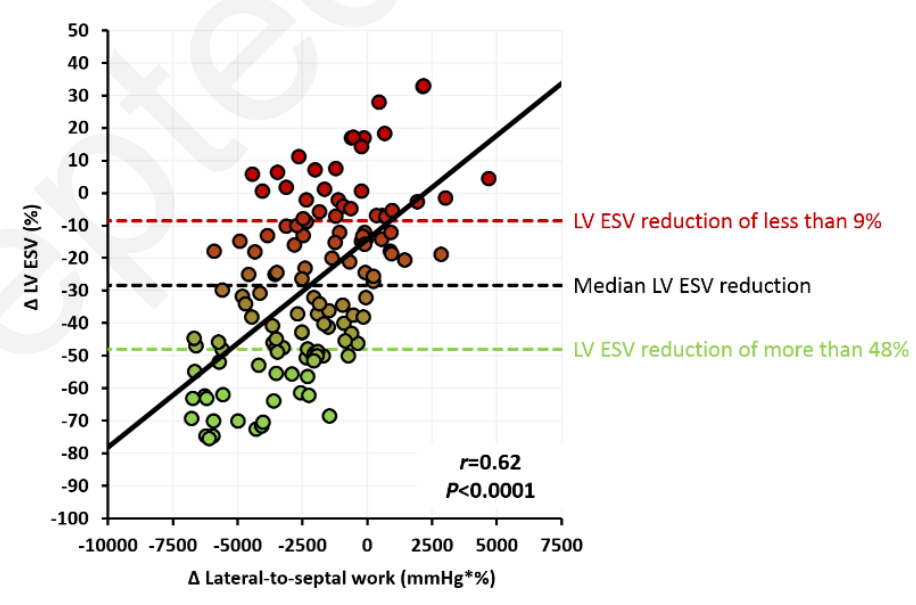

Figure 5: Correlation plot of $\Delta$ Lateral-to-septal work versus $\Delta$ LV ESV. Coloured dashed lines represent $\triangle$ LV ESV thresholds: patients with LV ESV reduction of less than $9 \%$ (red), with median LV ESV reduction (black), with LV ESV reduction of more than 48\% (green). 


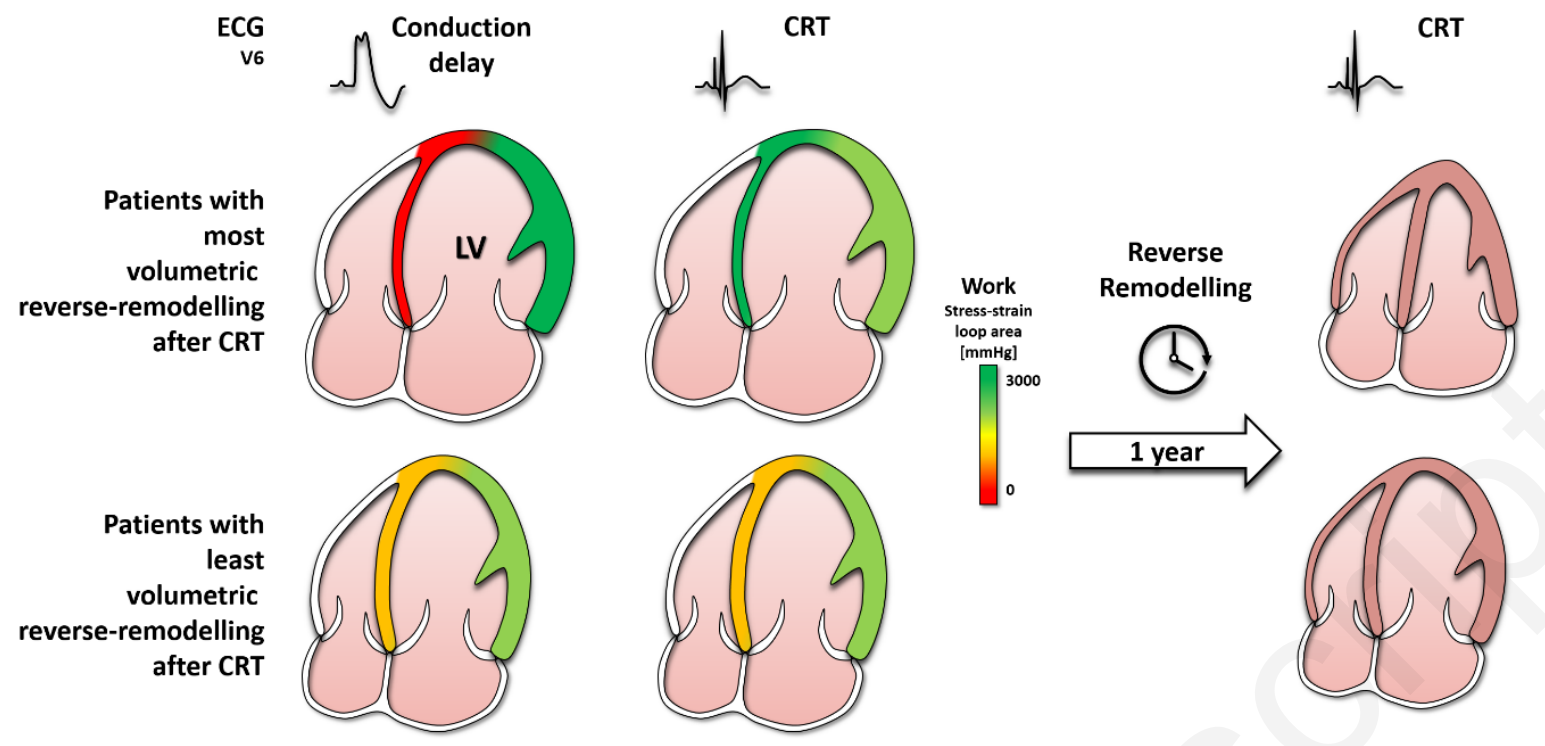

Figure 6: Reverse-remodelling follows loading. Top panels: in patients with most reverseremodelling after CRT, work is very inhomogeneously distributed across the LV before CRT, with low to negative work in the septum and high work in the lateral wall. Implanting a CRT causes a reversed pattern of inhomogeneity in work, with septal work being higher than the other LV walls. After time, these patients' heart demonstrates the highest reduction in LV volume. Lower panels: in patients with least reverse-remodelling after CRT, the septal and lateral wall show only a relatively small difference in work After CRT-implantation, no relevant changes in work are found. After time, these patients demonstrate no significant reduction in LV volume. 